\title{
First direct observation of time-reversal non-invariance in the neutral-kaon system
}

\section{CPLEAR Collaboration}

A. Angelopoulos ${ }^{\text {a }}$, A. Apostolakis ${ }^{\text {a }}$, E. Aslanides ${ }^{\mathrm{k}}$, G. Backenstoss ${ }^{\text {b }}$, P. Bargassa ${ }^{\mathrm{m}}$, O. Behnke ${ }^{\mathrm{q}}$, A. Benelli ${ }^{\mathrm{b}}$, V. Bertin ${ }^{\mathrm{k}}, \mathrm{F}$. Blanc ${ }^{\mathrm{g}, \mathrm{m}}, \mathrm{P}$. Bloch ${ }^{\mathrm{d}}$, P. Carlson ${ }^{\mathrm{o}}$, M. Carroll i, E. Cawley ${ }^{\text {i }, ~ S . ~ C h a r a l a m b o u s ~}{ }^{\mathrm{p}}$, M.B. Chertok ${ }^{\mathrm{c}}$, M. Danielsson ${ }^{\mathrm{o}}$, M. Dejardin ${ }^{n}$, J. Derre ${ }^{\mathrm{n}}$, A. Ealet ${ }^{\mathrm{k}}$, C. Eleftheriadis ${ }^{\mathrm{p}}$, L. Faravel ${ }^{\mathrm{g}}, \mathrm{W}$. Fetscher ${ }^{\mathrm{q}}$, M. Fidecaro ${ }^{\text {d }}$, A. Filipčič ${ }^{j}$, D. Francis ${ }^{\text {c }}$, J. Fry ${ }^{\text {i }, ~ E . ~ G a b a t h u l e r ~}{ }^{\text {i }}$, R. Gamet ${ }^{\text {i, }}$, H.-J. Gerber ${ }^{\text {q }}$, A. Go ${ }^{\text {d }}$, A. Haselden ${ }^{\text {i }, ~ P . J . ~ H a y m a n ~}{ }^{\text {i }, ~ F . ~ H e n r y-C o u a n n i e r ~}{ }^{k}$, R.W. Hollander ${ }^{\text {f }}$, K. Jon-And ${ }^{o}$, P.-R. Kettle ${ }^{\mathrm{m}}$, P. Kokkas ${ }^{\mathrm{d}}$, R. Kreuger ${ }^{\mathrm{f}}$, R. Le Gac ${ }^{\text {k }}$, F. Leimgruber ${ }^{\text {b }}$, I. Mandić ${ }^{\mathrm{j}}$, N. Manthos ${ }^{\text {h }}$, G. Marel ${ }^{\text {n }}$, M. Mikuž ${ }^{\mathrm{j}}$, J. Miller ${ }^{\text {c }}$, F. Montanet ${ }^{\mathrm{k}}$, A. Muller ${ }^{\mathrm{n}}$, T. Nakada ${ }^{\mathrm{m}}$, B. Pagels ${ }^{\mathrm{q}}$, I. Papadopoulos ${ }^{\text {p }, ~ P . ~ P a v l o p o u l o s ~}{ }^{\mathrm{b}}$, A. Policarpo ${ }^{\mathrm{e}}$, G. Polivka ${ }^{\mathrm{b}}$, R. Rickenbach ${ }^{\mathrm{b}}$, B.L. Roberts ${ }^{\text {c }}$, T. Ruf ${ }^{\text {d }}$, C. Santoni ${ }^{\text {b }}$, M. Schäfer ${ }^{\text {q }}$, L.A. Schaller ${ }^{\mathrm{g}}$, T. Schietinger ${ }^{\mathrm{b}}$, A. Schopper ${ }^{\mathrm{d}}$, L. Tauscher ${ }^{\mathrm{b}}$, C. Thibault ${ }^{1}$, F. Touchard ${ }^{\mathrm{k}}$, C. Touramanis ${ }^{\text {i }}$, C.W.E. Van Eijk ${ }^{\mathrm{f}}$, S. Vlachos ${ }^{\mathrm{b}}$, P. Weber ${ }^{\mathrm{q}}$, O. Wigger ${ }^{\mathrm{m}}$, M. Wolter ${ }^{\mathrm{q}}$, D. Zavrtanik ${ }^{\mathrm{j}}$, D. Zimmerman ${ }^{\mathrm{c}}$

\author{
${ }^{\mathrm{a}}$ University of Athens, Greece \\ ${ }^{\mathrm{b}}$ University of Basle, Switzerland \\ c Boston University, USA \\ ${ }^{\mathrm{d}}$ CERN, Geneva, Switzerland \\ ${ }^{\mathrm{e}}$ LIP and University of Coimbra, Portugal \\ ${ }^{\mathrm{f}}$ Delft University of Technology, Netherlands \\ ${ }^{\mathrm{g}}$ University of Fribourg, Switzerland \\ ${ }^{\mathrm{h}}$ University of Ioannina, Greece \\ ${ }^{\mathrm{i}}$ University of Liverpool, $U K$ \\ ${ }^{\mathrm{j}}$ J. Stefan Inst. and Phys. Dep., University of Ljubljana, Slovenia \\ ${ }^{\mathrm{k}}$ CPPM, IN2P3-CNRS et Université d'Aix-Marseille II, France \\ ${ }^{1}$ CSNSM, IN2P3-CNRS, Orsay, France \\ ${ }^{\mathrm{m}}$ Paul Scherrer Institut (PSI), Switzerland \\ ${ }^{\mathrm{n}}$ CEA, DSM / DAPNIA, CE-Saclay, France \\ ${ }^{\circ}$ Royal Institute of Technology, Stockholm, Sweden
}


${ }^{\mathrm{p}}$ University of Thessaloniki, Greece

${ }^{\text {q }}$ ETH-IPP Zürich, Switzerland

Received 19 October 1998

Editor: L. Montanet

\section{Abstract}

We report on the first observation of time-reversal symmetry violation through a comparison of the probabilities of $\overline{\mathrm{K}}^{0}$ transforming into $\mathrm{K}^{0}$ and $\mathrm{K}^{0}$ into $\overline{\mathrm{K}}^{0}$ as a function of the neutral-kaon eigentime $t$. The comparison is based on the analysis of the neutral-kaon semileptonic decays recorded in the CPLEAR experiment. There, the strangeness of the neutral kaon at time $t=0$ was tagged by the kaon charge in the reaction $\mathrm{p} \overline{\mathrm{p}} \rightarrow \mathrm{K}^{ \pm} \pi^{\mp} \mathrm{K}^{0}\left(\overline{\mathrm{K}}^{0}\right)$ at rest, whereas the strangeness of the kaon at the decay time $t=\tau$ was tagged by the lepton charge in the final state. An average decay-rate asymmetry

$$
\left\langle\frac{R\left(\overline{\mathrm{K}}_{t=0}^{0} \rightarrow \mathrm{e}^{+} \pi^{-} v_{t=\tau}\right)-R\left(\mathrm{~K}_{t=0}^{0} \rightarrow \mathrm{e}^{-} \pi^{+} \bar{v}_{t=\tau}\right)}{R\left(\overline{\mathrm{K}}_{t=0}^{0} \rightarrow \mathrm{e}^{+} \pi^{-} v_{t=\tau}\right)+R\left(\mathrm{~K}_{t=0}^{0} \rightarrow \mathrm{e}^{-} \pi^{+} \bar{v}_{t=\tau}\right)}\right\rangle=\left(6.6 \pm 1.3_{\text {stat }} \pm 1.0_{\text {syst }}\right) \times 10^{-3}
$$

was measured over the interval $1 \tau_{\mathrm{S}}<\tau<20 \tau_{\mathrm{S}}$, thus leading to evidence for time-reversal non-invariance. (C) 1998 Elsevier Science B.V. All rights reserved.

\section{Introduction}

Since weak interactions do not conserve strangeness, a $\mathrm{K}^{0}$ meson can transform into a $\overline{\mathrm{K}}^{0}$ in the course of time, and vice-versa, a $\overline{\mathrm{K}}^{0}$ can transform into a $\mathrm{K}^{0}$. Time-reversal (T) invariance, or microscopic reversibility, would require all details of the second process to be deducible from the first; in particular, the probability $(\mathscr{P})$ that a $\mathrm{K}^{0}(t=0)$ is observed as a $\overline{\mathrm{K}}^{0}$ at time $\tau$ should be equal to the probability that a $\overline{\mathrm{K}}^{0}(t=0)$ is observed as a $\mathrm{K}^{0}$ at the same time $\tau$ [1]. Any difference between these two probabilities is a signal for $\mathrm{T}$ violation and can be measured through the time-reversal asymmetry

$$
\frac{\mathscr{P}\left(\overline{\mathrm{K}}^{0} \rightarrow \mathrm{K}^{0}\right)-\mathscr{P}\left(\mathrm{K}^{0} \rightarrow \overline{\mathrm{K}}^{0}\right)}{\mathscr{P}\left(\overline{\mathrm{K}}^{0} \rightarrow \mathrm{K}^{0}\right)+\mathscr{P}\left(\mathrm{K}^{0} \rightarrow \overline{\mathrm{K}}^{0}\right)} .
$$

Experimentally this requires knowledge of the strangeness of the neutral kaon at two different times of its life.

A measurement of this asymmetry has become possible with the CPLEAR experiment, which produced $\mathrm{K}^{0} \mathrm{~s}$ and $\overline{\mathrm{K}}^{0} \mathrm{~s}$ through the strong interactions

$$
\mathrm{p} \overline{\mathrm{p}} \rightarrow\left\{\begin{array}{l}
\mathrm{K}^{-} \pi^{+} \mathrm{K}^{0} \\
\mathrm{~K}^{+} \pi^{-} \overline{\mathrm{K}}^{0}
\end{array}\right.
$$

enabling the initial strangeness of the neutral kaon to be tagged by the charge of the accompanying charged kaon. To tag the strangeness of the kaon at the moment of its decay we use semileptonic decays: positive lepton charge is associated to a $\mathrm{K}^{0}$ and negative lepton charge to a $\overline{\mathrm{K}}^{0}$. We measure, as a function of time, the decay-rate asymmetry

$$
\frac{R\left(\overline{\mathrm{K}}_{t=0}^{0} \rightarrow \mathrm{e}^{+} \pi^{-} \nu_{t=\tau}\right)-R\left(\mathrm{~K}_{t=0}^{0} \rightarrow \mathrm{e}^{-} \pi^{+} \bar{v}_{t=\tau}\right)}{R\left(\overline{\mathrm{K}}_{t=0}^{0} \rightarrow \mathrm{e}^{+} \pi^{-} \nu_{t=\tau}\right)+R\left(\mathrm{~K}_{t=0}^{0} \rightarrow \mathrm{e}^{-} \pi^{+} \bar{v}_{t=\tau}\right)}
$$

In the limit of CPT symmetry in the semileptonic decay process and of the validity of the $\Delta S=\Delta Q$ rule, this asymmetry is identical with the time-reversal asymmetry given in (1). 
In the past, phenomenological studies based on the Bell-Steinberger relation have concluded that CP violation in the neutral-kaon system is dominantly accompanied by CPT invariance and $\mathrm{T}$ violation [2]. However, owing to its importance, the time-reversal symmetry deserved to be tested more directly. With the experiment presented here we report for the first time a direct observation of a T-odd asymmetry.

\section{The experiment}

Full details of the design, operation and performance of the CPLEAR detector can be found in Ref. [3]. Here only a short overview of the detector is presented.

The CPLEAR detector had a cylindrical geometry and was mounted inside a solenoid magnet that produced a field of $0.44 \mathrm{~T}$. It allowed the detection of neutral-kaon decays in the time range $0<\tau<20 \tau_{\mathrm{S}}\left(\tau_{\mathrm{S}}\right.$ is the $\mathrm{K}_{\mathrm{S}}$ mean life). The $200 \mathrm{MeV} / c$ antiprotons from the Low Energy Antiproton Ring (LEAR) of CERN stopped and annihilated inside a gaseous hydrogen target at the centre of the detector. Going radially outwards from the target, the detector consisted of a tracking device (two multiwire proportional chambers, six drift chambers and two layers of streamer tubes), a scintillator-Cherenkov-scintillator sandwich (S1-CE-S2) for particle identification, and a lead/gas-sampling electromagnetic calorimeter. A multi-level trigger system provided fast event selection. The trigger was based on particle identification, event kinematics and shower counting.

\section{Event selection}

The desired $\mathrm{p} \overline{\mathrm{p}}$ annihilations followed by the decay of the neutral kaon into e $\pi \nu$ are first selected by requiring that the events have four charged tracks and zero total charge as well as a good reconstruction quality for each track and vertex, and by identifying one of the decay tracks as an electron or a positron.

The lepton identification is performed with a Neural Network (NN) algorithm [4,5]. The inputs to the NN algorithm are the momentum of the particle, the energy loss in the two scintillators (S1, S2), the number of photoelectrons in the Cherenkov counter and the time of flight from the decay vertex to the first scintillator (S1).

The Neural Network was trained using pure samples of electrons and pions. The pion sample consisted of pions from neutral-kaon decays to $\pi^{+} \pi^{-}$. The electron sample was obtained with a special trigger which selected $\mathrm{p} \overline{\mathrm{p}}$ annihilations to $\pi^{+} \pi^{-} \pi^{0}$. The $\pi^{0}$ decayed into two photons, one of which in turn converted into an electron-positron pair in the detector material.

The electron identification efficiency, obtained requiring that less than $2 \%$ of pions be misidentified as electrons, is on the average $85 \%$. No attempt is made to identify muons with the NN. The probability to identify a muon as an electron, and thus include a muon event in the electron signal, is $\approx 15 \%$.

Finally, the method of constrained fits is used:

- 1C-fit. The hypothesis of the $\mathrm{p} \overline{\mathrm{p}} \rightarrow \mathrm{K}^{ \pm} \pi^{\mp} \mathrm{K}^{0}\left(\overline{\mathrm{K}}^{0}\right)$ channel is assumed for the annihilation event. The event is kept only if the fit yields a probability greater than $10 \%$.

- 6C-fit. This fit includes kinematic constraints (energy-momentum conservation under the semileptonic decay hypothesis) and geometric constraints (the alignment of the $\mathrm{K}^{0}$ momentum vector with the line joining the production with the decay vertex, and a good $z$-intersection of the tracks at the two vertices). The event is kept only if the probability is greater than $5 \%$.

- 4C-fit. This fit is performed in order to reduce the $\pi^{+} \pi^{-}$background that comes from the decays $\mathrm{K}^{0}\left(\overline{\mathrm{K}}^{0}\right) \rightarrow \pi^{+} \pi^{-}$. The events are passed through the kinematic constrained fit with the $\pi^{+} \pi^{-}$decay hypothesis. If the probability to fit the hypothesis is greater than $10 \%$, the event is rejected.

The fitted momenta and vertices resulting from the $6 \mathrm{C}$-fit determine the neutral-kaon lifetime with a precision which ranges from $0.05 \tau_{\mathrm{S}}$ in the short lifetime region to $0.2-0.3 \tau_{\mathrm{S}}$ in the long one.

A total of $1.3 \times 10^{6} \mathrm{e} \pi \nu$ events, with measured decay time $\tau \geq 1 \tau_{\mathrm{S}}$, survive the above selection. 


\section{Analysis}

A number of factors that can introduce a distortion of the rates must be considered: detection efficiencies, regeneration effects, background estimation and decay-time resolution. The latter was taken into account during the fit (Section 6), by folding the decay-time resolutions [3] in the fitting function.

\subsection{Normalization of $\mathrm{K}^{0}$ and $\overline{\mathrm{K}}^{0}$ rates}

Using an asymmetry has the advantage that the detection efficiencies common to the two processes being compared cancel. Differences in the geometrical acceptances are compensated to first order by frequently reversing the magnetic field. However, different detection probabilities for the charged kaons, pions and electrons used for tagging the strangeness of the neutral kaon at production and decay times lead to different corrections for each event sample. The corrections are performed on an event-by-event basis via the two normalization factors which are important for the decay-rate asymmetry given in (2):

- $\xi=\epsilon\left(\mathrm{K}^{+} \pi^{-}\right) / \epsilon\left(\mathrm{K}^{-} \pi^{+}\right)$, where the efficiencies involved, $\epsilon(\mathrm{K} \pi)$, are those of the charged particles at the production vertex (primary vertex normalization);

- $\eta=\epsilon\left(\pi^{+} \mathrm{e}^{-}\right) / \epsilon\left(\pi^{-} \mathrm{e}^{+}\right)$, which takes into account the different detection efficiencies, $\epsilon(\pi \mathrm{e})$, for the particles in the two final states (secondary vertex normalization).

\subsubsection{Primary vertex normalization}

The primary vertex normalization factor $\xi$ differs from unity because of the different strong interaction cross-sections of oppositely charged kaons and pions. These differences are parametrized in terms of transverse and longitudinal momentum of the charged kaon, $p_{\mathrm{t}}\left(\mathrm{K}^{ \pm}\right)$and $p_{z}\left(\mathrm{~K}^{ \pm}\right)$, and of the momentum of the primary pion, $p\left(\pi^{\mp}\right), \xi=\xi\left(p_{\mathrm{t}}\left(\mathrm{K}^{ \pm}\right), p_{z}\left(\mathrm{~K}^{ \pm}\right), p\left(\pi^{\mp}\right)\right)$.

The primary normalization factor $\xi$ is independent of the final state into which the neutral kaon decays. We obtain it with high precision from our data set of $\pi^{+} \pi^{-}$decays where more and better constrained events are available [6]. We select events with decay times between 1 and $4 \tau_{\mathrm{S}}$ and calculate the normalization factor $\xi$ by building the ratio of observed $\mathrm{K}^{0}$ to $\overline{\mathrm{K}}^{0}$ events. This ratio must follow the neutral-kaon time evolution, and, in the time interval considered, can be approximated as

$$
\frac{\xi N\left(\mathrm{~K}_{t=0}^{0} \rightarrow \pi^{+} \pi_{t=\tau}^{-}\right)}{N\left(\overline{\mathrm{K}}_{t=0}^{0} \rightarrow \pi^{+} \pi_{t=\tau}^{-}\right)}=\left(1-4 \operatorname{Re}\left(\epsilon_{\mathrm{L}}\right)\right) \times\left(1+4\left|\eta_{+-}\right| \cos \left(\Delta m \tau-\phi_{+-}\right) \mathrm{e}^{\frac{1}{2} \Gamma_{\mathrm{S}} \tau}\right) .
$$

The oscillating factor on the right-hand side of Eq. (3) depends on the neutral-kaon parameters $\eta_{+-}, \Delta m$ and $\Gamma_{\mathrm{S}} \equiv 1 / \tau_{\mathrm{S}}$, which we take from [7], and, as a result, is known with a precision of $\approx 1 \times 10^{-4}$. The quantitiy $\operatorname{Re}\left(\epsilon_{\mathrm{L}}\right)$ is taken from the semileptonic charge asymmetry $\delta_{l}, 4 \operatorname{Re}\left(\epsilon_{\mathrm{L}}\right)$ being equal to $2 \delta_{l}$ in the limit of CPT symmetry in semileptonic decays. Experimentally, the charge asymmetry is [7]

$$
\delta_{l}=(3.27 \pm 0.12) \times 10^{-3} .
$$

The average of $\xi$ over all pion and kaon momenta is $\langle\xi\rangle=1.12023 \pm 0.00043$ where the main contribution to the error is the statistical error of the two-pion decays.

\subsubsection{Secondary vertex normalization}

The secondary vertex normalization factor, $\eta$, can be factorised as $\eta\left(p_{\pi}, p_{\mathrm{e}}\right)=\left[\epsilon\left(\pi^{+}\right) / \epsilon\left(\pi^{-}\right)\right] \times$ $\left[\epsilon\left(\mathrm{e}^{-}\right) / \epsilon\left(\mathrm{e}^{+}\right)\right]$, and is measured as a function of the momentum of the decay pion $\left(p_{\pi}\right)$ and electron $\left(p_{\mathrm{e}}\right)$.

There are several contributions which may cause $\eta$ to differ from unity. We have considered different probabilities for $\pi^{+}$and $\pi^{-}$and for $\mathrm{e}^{+}$and $\mathrm{e}^{-}$to be detected in the scintillator (S1), curvature-dependent trigger efficiency to recognize a track, and $\mathrm{NN}$ selection-criteria efficiencies. 
Pure and unbiased samples of pions and electrons were used to study the difference in the efficiencies. A sample of $\mathrm{e}^{+} \mathrm{e}^{-}$pairs has been selected from $\gamma$ conversion data $\left(\mathrm{K}^{0} \rightarrow 2 \pi^{0}, \pi^{0} \rightarrow 2 \gamma, \gamma \rightarrow \mathrm{e}^{+} \mathrm{e}^{-}\right)$, and a sample of $\pi^{+}$and $\pi^{-}$tracks has been selected from minimum-bias data. The different probabilities to be detected in the scintillator (S1), a fundamental input for e $/ \pi$ identification, have been extracted from both these data samples. The minimum-bias data have been used to parametrize any additional difference between opposite-charge tracks caused by the trigger. The value of $\eta$, averaged over the particle momenta, is $\langle\eta\rangle=1.014 \pm 0.002$.

In order to cross check our secondary vertex normalization, we can determine the charge asymmetry $\delta_{l}$ from our data. This asymmetry is directly proportional to $\eta$ and any variation of $\eta$ propagates to $\delta_{l}$ as $\Delta\left(\delta_{l}\right)=\frac{1}{2} \Delta(\langle\eta\rangle)$. We obtain $\delta_{l}=(2.8 \pm 1.3) \times 10^{-3}$, consistent with the world average value given in Eq. (4).

\subsection{Regeneration}

$\mathrm{K}^{0}$ and $\overline{\mathrm{K}}^{0}$ are subject to regeneration arising from the forward scattering in the material of the detector. The CPLEAR experiment has dedicated the final year of data-taking to measure the regeneration amplitudes $\Delta f=f(0)-\bar{f}(0)$. The results are reported in Ref. [8]. Regeneration corrections are calculated and applied on an event-by-event basis, depending on the momentum of the neutral kaon and on the positions of its production and decay vertices within the detector. This correction results in a positive shift of the asymmetry of $0.3 \times 10^{-3}$.

\subsection{Background}

From the Monte Carlo simulation we obtain the background to signal ratio for the different background channels as a function of the decay time [5]. We have considered contributions from neutral-kaon decays, namely $\pi \mathrm{e} \nu, \pi \mu \nu, \pi^{+} \pi^{-}, \pi^{+} \pi^{-} \pi^{0}$ and $\pi^{0} \pi^{0}$ with one Dalitz decay or one converted photon. The main background source consists of neutral-kaon two-pion decays and is concentrated at early decay times, while at late decay times there are contributions from $\pi \mathrm{e} \nu$ decays where the electron and pion assignments are exchanged, and from $\pi^{+} \pi^{-} \pi^{0}$ decays. Their levels relative to the signal remain below $1 \%$.

\subsection{The asymmetry $A_{\mathrm{T}}^{\exp }$}

After regeneration and background correction, the number of $\mathrm{K}^{0}$ events is normalized to the number of $\overline{\mathrm{K}}^{0}$ with the factor $\xi$, and the number of $\left(\mathrm{e}^{+} \pi^{-}\right)$decays to the number of $\left(\mathrm{e}^{-} \pi^{+}\right)$decays with the factor $\eta$. This leads to the experimental asymmetry

$$
A_{\mathrm{T}}^{\exp }(\tau)=\frac{\eta N\left(\overline{\mathrm{K}}_{t=0}^{0} \rightarrow \mathrm{e}^{+} \pi^{-} \nu_{t=\tau}\right)-\xi N\left(\mathrm{~K}_{t=0}^{0} \rightarrow \mathrm{e}^{-} \pi^{+} \bar{v}_{t=\tau}\right)}{\eta N\left(\overline{\mathrm{K}}_{t=0}^{0} \rightarrow \mathrm{e}^{+} \pi^{-} \nu_{t=\tau}\right)+\xi N\left(\mathrm{~K}_{t=0}^{0} \rightarrow \mathrm{e}^{-} \pi^{+} \bar{v}_{t=\tau}\right)},
$$

where $N$ stands for the observed number of initial $\overline{\mathrm{K}}^{0}$ accompanied by a decay $\mathrm{e}^{+}$or the observed number of $\mathrm{K}^{0}$ accompanied by an $\mathrm{e}^{-}$, as indicated in brackets. The total number of events entering in this asymmetry is $6.4 \times 10^{5}$.

The asymmetry is shown in Fig. 1 for a decay-time interval $1 \tau_{\mathrm{S}} \leq \tau \leq 20 \tau_{\mathrm{S}}$. The data points scatter around a positive and constant offset from zero, the average being

$$
\left\langle A_{\mathrm{T}}^{\exp }\right\rangle_{(1-20) \tau_{\mathrm{S}}}=(6.6 \pm 1.3) \times 10^{-3},
$$

with $\chi^{2} /$ d.o.f. $=0.84$. 


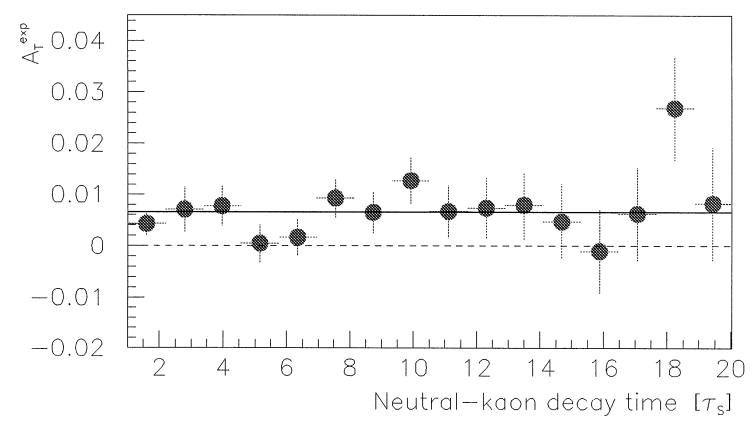

Fig. 1. The asymmetry $A_{\mathrm{T}}^{\text {exp }}$ versus the neutral-kaon decay time (in units of $\tau_{\mathrm{S}}$ ). The solid line represents the fitted average $\left\langle A_{\mathrm{T}}^{\exp }\right\rangle$.

This is an evidence for $\mathrm{T}$ violation. However, some uncertainty may result from the strangeness tagging at the neutral kaon decay, due to a possible violation of the $\Delta S=\Delta Q$ rule, and from a possible CPT violation in semileptonic decays. For a thorough analysis of such effects we need to enter into the phenomenology of the neutral kaons.

\section{Phenomenology of the method}

The time evolution and decay of the neutral kaons can be parametrized in terms of different, mostly equivalent, mixing and decay parameters. We briefly outline the parametrization used in our analysis $[9,10]$.

The two parameters which describe the neutral-kaon mixing, are, respectively, the $\mathrm{T}$ and CPT violation parameters:

$$
\epsilon=\frac{\Lambda_{\overline{\mathrm{K}}^{0}, \mathrm{~K}^{0}}-\Lambda_{\mathrm{K}^{0}, \overline{\mathrm{K}}^{0}}}{2\left(\lambda_{\mathrm{L}}-\lambda_{\mathrm{S}}\right)} \quad \text { and } \quad \delta=\frac{\Lambda_{\overline{\mathrm{K}}^{0}, \overline{\mathrm{K}}^{0}}-\Lambda_{\mathrm{K}^{0}, \mathrm{~K}^{0}}}{2\left(\lambda_{\mathrm{L}}-\lambda_{\mathrm{S}}\right)} .
$$

Here, $\Lambda_{i j}$ are the elements and $\lambda_{\mathrm{L}, \mathrm{S}}$ the eigenvalues of the effective Hamiltonian $\Lambda: \lambda_{\mathrm{L}, \mathrm{S}}=m_{\mathrm{L}, \mathrm{S}}-\frac{\mathrm{i}}{2} \Gamma_{\mathrm{L}, \mathrm{S}}$, where $m_{\mathrm{L}, \mathrm{S}}$ and $\Gamma_{\mathrm{L}, \mathrm{S}}$ are the masses and decay widths for the $\mathrm{K}_{\mathrm{L}}$ and $\mathrm{K}_{\mathrm{S}}$ states, $\Delta m=m_{\mathrm{L}}-m_{\mathrm{S}}$ and $\Delta \Gamma=\Gamma_{\mathrm{S}}-\Gamma_{\mathrm{L}}$. Note that $\epsilon$ is $\mathrm{T}$ violating by construction. The $\mathrm{K}_{\mathrm{L}}$ mixing parameter is defined as $\epsilon_{\mathrm{L}}=\epsilon-\delta$.

The semileptonic decay amplitudes can be written as

$$
\begin{array}{ll}
\left\langle\mathrm{e}^{+} \pi^{-} \nu|\Lambda| \mathrm{K}^{0}\right\rangle=a+b, & \left\langle\mathrm{e}^{-} \pi^{+} \bar{\nu}|\Lambda| \overline{\mathrm{K}}^{0}\right\rangle=a^{*}-b^{*}, \\
\left\langle\mathrm{e}^{-} \pi^{+} \bar{\nu}|\Lambda| \mathrm{K}^{0}\right\rangle=c+d, & \left\langle\mathrm{e}^{+} \pi^{-} \nu|\Lambda| \overline{\mathrm{K}}^{0}\right\rangle=c^{*}-d^{*} .
\end{array}
$$

The amplitudes $b$ and $d$ are CPT violating, $c$ and $d$ describe possible violations of the $\Delta S=\Delta Q$ rule, and the imaginary parts are all $\mathrm{T}$ violating. The quantities

$$
x=\frac{c^{*}-d^{*}}{a+b} \text { and } \bar{x}=\frac{c^{*}+d^{*}}{a-b}
$$

describe the violation of the $\Delta S=\Delta Q$ rule in decays into positive and negative leptons, respectively, while

$$
y=-\frac{b}{a}
$$

describes CPT violation in semileptonic decays in the case where the $\Delta S=\Delta Q$ rule holds. The parameters $x_{+}=(x+\bar{x}) / 2$ and $x_{-}=(x-\bar{x}) / 2$ describe the violation of the $\Delta S=\Delta Q$ rule in CPT-conserving and CPT-violating amplitudes, respectively. We assume $x, \bar{x}$ and $y \ll 1$. 
The semileptonic decay rates depend on the strangeness of the kaon $\left(\mathrm{K}^{0}\right.$ or $\left.\overline{\mathrm{K}}^{0}\right)$ at the production time, $t=0$, and on the charge of the decay lepton $\left(\mathrm{e}^{+}\right.$or $\left.\mathrm{e}^{-}\right)$:

$$
\begin{array}{ll}
R_{+}(\tau) \equiv R\left[\mathrm{~K}_{t=0}^{0} \rightarrow \mathrm{e}^{+} \pi^{-} v_{t=\tau}\right], & \bar{R}_{-}(\tau) \equiv R\left[\overline{\mathrm{K}}_{t=0}^{0} \rightarrow \mathrm{e}^{-} \pi^{+} \bar{v}_{t=\tau}\right], \\
R_{-}(\tau) \equiv R\left[\mathrm{~K}_{t=0}^{0} \rightarrow \mathrm{e}^{-} \pi^{+} \bar{v}_{t=\tau}\right], & \bar{R}_{+}(\tau) \equiv R\left[\overline{\mathrm{K}}_{t=0}^{0} \rightarrow \mathrm{e}^{+} \pi^{-} v_{t=\tau}\right] .
\end{array}
$$

In the limit of validity of the $\Delta S=\Delta Q$ rule, the rates $R_{-}(\tau)$ and $\bar{R}_{+}(\tau)$ measure the probability of an initial $\mathrm{K}^{0}$ being a $\overline{\mathrm{K}}^{0}$ at the decay time and of the time-reversed process, respectively, and are thus the rates that enter in the time-reversal asymmetry. These rates can be written as a function of the neutral-kaon decay time, $\tau$, and of the parameters $\operatorname{Re}(\epsilon), \operatorname{Re}(y), x$ and $\bar{x}$ :

$$
\begin{aligned}
R_{-}(\tau)= & \frac{|a|^{2}}{4}\left([1+2 \operatorname{Re}(\bar{x})-4 \operatorname{Re}(\epsilon)+2 \operatorname{Re}(y)] \mathrm{e}^{-\Gamma_{\mathrm{S}} \tau}+[1-2 \operatorname{Re}(\bar{x}))-4 \operatorname{Re}(\epsilon)+2 \operatorname{Re}(y)\right] \mathrm{e}^{-\Gamma_{\mathrm{L}} \tau} \\
& \left.-[2(1-4 \operatorname{Re}(\epsilon)+2 \operatorname{Re}(y)) \cos (\Delta m \tau)+4 \operatorname{Im}(\bar{x}) \sin (\Delta m \tau)] \mathrm{e}^{-\frac{1}{2}\left(\Gamma_{\mathrm{S}}+\Gamma_{\mathrm{L}}\right) \tau}\right) \\
\bar{R}_{+}(\tau)= & \frac{|a|^{2}}{4}\left([1+2 \operatorname{Re}(x)+4 \operatorname{Re}(\epsilon)-2 \operatorname{Re}(y)] \mathrm{e}^{-\Gamma_{\mathrm{S}} \tau}+[1-2 \operatorname{Re}(x)+4 \operatorname{Re}(\epsilon)-2 \operatorname{Re}(y)] \mathrm{e}^{-\Gamma_{\mathrm{L}} \tau}\right. \\
& \left.-[2(1+4 \operatorname{Re}(\epsilon)-2 \operatorname{Re}(y)) \cos (\Delta m \tau)-4 \operatorname{Im}(x) \sin (\Delta m \tau)] \mathrm{e}^{-\frac{1}{2}\left(\Gamma_{\mathrm{S}}+\Gamma_{\mathrm{L}}\right) \tau}\right)
\end{aligned}
$$

We neglect second-order terms, which only play a role for very short lifetime $\left(\tau \ll 1 \tau_{\mathrm{S}}\right)$. The parameters $\operatorname{Re}(y), \operatorname{Re}\left(x_{-}\right)$also enter the determination of the parameter $\operatorname{Re}\left(\epsilon_{\mathrm{L}}\right)$ from the charge asymmetry $\delta_{l}$,

$$
\operatorname{Re}\left(\epsilon_{\mathrm{L}}\right)=\frac{1}{2}\left(\delta_{l}+2 \operatorname{Re}(y)+2 \operatorname{Re}\left(x_{-}\right)\right) .
$$

\section{Fit results}

The rates in Eqs. (9b) and (9c) are linked to the measurement through the normalised rates $R_{-} \propto \xi N\left(\mathrm{~K}_{t=0}^{0}\right.$ $\left.\rightarrow \mathrm{e}^{-} \pi^{+} \bar{v}_{t=\tau}\right)$ and $\bar{R}_{+} \propto \eta N\left(\overline{\mathrm{K}}_{t=0}^{0} \rightarrow \mathrm{e}^{+} \pi^{-} \nu_{t=\tau}\right)$. However, the normalisation procedure of Eq. (3) using $2 \delta_{l}=4 \operatorname{Re}\left(\epsilon_{\mathrm{L}}\right)$ has to be corrected for the two CPT-violating extra terms. In fact this corresponds to $\xi N\left(\mathrm{~K}_{t=0}^{0} \rightarrow \mathrm{e}^{-} \pi^{+} \bar{v}_{t=\tau}\right) \propto\left(1+4 \operatorname{Re}\left(x_{-}\right)+4 \operatorname{Re}(y)\right) R_{-}$. The $A_{\mathrm{T}}^{\exp }$ asymmetry can be expressed by

$$
\begin{gathered}
A_{\mathrm{T}}^{\mathrm{exp}}(\tau) \approx \frac{\bar{R}_{+}(\tau)-R_{-}(\tau)}{\bar{R}_{+}(\tau)+R_{-}(\tau)}-2 \operatorname{Re}(y)-2 \operatorname{Re}\left(x_{-}\right)=4\left(\operatorname{Re}(\epsilon-y)-\operatorname{Re}\left(x_{-}\right)\right) \\
+2 \frac{\operatorname{Re}\left(x_{-}\right)\left(\mathrm{e}^{-\frac{1}{2} \Delta \Gamma \tau}+\cos (\Delta m \tau)\right)+\operatorname{Im}\left(x_{+}\right) \sin (\Delta m \tau)}{\cosh \left(\frac{1}{2} \Delta \Gamma \tau\right)-\cos (\Delta m \tau)},
\end{gathered}
$$

where again we neglect second-order terms in the parameters. For long lifetimes this asymmetry is constant, $A_{\mathrm{T}}^{\exp }=4\left(\operatorname{Re}(\epsilon-y)-\operatorname{Re}\left(x_{-}\right)\right)$.

In the following analysis we assume CPT invariance in the semileptonic decay amplitudes, $(\operatorname{Re}(y)=0$ and $\left.x_{-}=0\right)$. This assumption will be discussed in a forthcoming paper. We allow for a possible violation of the 
$\Delta S=\Delta Q$ rule $\left(x_{+} \neq 0\right)$. The fitting procedure then contains only two parameters, $\operatorname{Re}(\epsilon)$ and $\operatorname{Im}\left(x_{+}\right)$, both $\mathrm{T}$ violating; $\Delta m, \Gamma_{\mathrm{S}}$ and $\Gamma_{\mathrm{L}}$ are taken as the world averages [7]. The result of the fit is

$$
\begin{aligned}
& 4 \operatorname{Re}(\epsilon)=(6.2 \pm 1.4) \times 10^{-3}, \\
& \operatorname{Im}\left(x_{+}\right)=(1.2 \pm 1.9) \times 10^{-3},
\end{aligned}
$$

with $\chi^{2} /$ d.o.f. $=0.84$. The errors are statistical only. The correlation coefficient between $4 \operatorname{Re}(\epsilon)$ and $\operatorname{Im}\left(x_{+}\right)$ is 0.46 .

We observe clear evidence for $\mathrm{T}$ violation in the neutral-kaon mixing. $\operatorname{Im}\left(x_{+}\right)$is compatible with zero. Thus, no $\mathrm{T}$ violation is observed in the semileptonic decay amplitude which violates the $\Delta S=\Delta Q$ rule, should this amplitude be different from zero. We note that $\operatorname{Im}\left(x_{+}\right)$is given by the value of the asymmetry for short lifetimes while $4 \operatorname{Re}(\epsilon)$ is determined by the long lifetime values. As a result the average $\left\langle A_{\mathrm{T}}^{\exp }\right\rangle$ between 1 and $20 \tau_{\mathrm{S}}$ is essentially equal to $4 \operatorname{Re}(\epsilon)$.

\section{Systematic errors}

We have investigated the following sources of systematic errors in the measurement of $\left\langle A_{\mathrm{T}}^{\exp }\right\rangle$ :

- background level and background asymmetry,

- normalization factors,

- decay-time resolution,

- regeneration correction.

A summary of the systematic errors for the different parameters is reported in Table 1 . Note that the systematic errors on $\left\langle A_{\mathrm{T}}^{\exp }\right\rangle$ applies as well to $4 \operatorname{Re}(\epsilon)$ for the case of the two parameters fit.

The determination of the relative acceptances for the various background contributions is made using a Monte Carlo simulation, which is known to reproduce the data to better than $10 \%$. A change of $10 \%$ in the relative acceptances for the various background contributions results in a systematic uncertainty in $\left\langle A_{\mathrm{T}}^{\exp }\right\rangle$ of $\pm 0.03 \times 10^{-3}$.

An additional source of systematic error arises from the charge asymmetry in the background, owing to the different probabilities for a $\pi^{+}$and a $\pi^{-}$to be identified as a positron and an electron, respectively. The level of this asymmetry has been determined using pions selected from minimum-bias events and is found to be $(3 \pm 1) \%$, leading to an uncertainty in $\left\langle A_{\mathrm{T}}^{\exp }\right\rangle$ of $\pm 0.02 \times 10^{-3}$.

In order to estimate the effect of an error in the normalization functions, $\xi$ and $\eta$, we change the value of the average normalization in the fit procedure by one standard deviation. The parameters $\xi$ and $\eta$ are measured to an accuracy of $\pm 4.3 \times 10^{-4}$ and $\pm 2.0 \times 10^{-3}$ resulting in a systematic error on $\left\langle A_{\mathrm{T}}^{\exp }\right\rangle$ of $\pm 0.2 \times 10^{-3}$ and

Table 1

Summary of systematic errors

\begin{tabular}{llll}
\hline Source & Known precision & $\left\langle A_{\mathrm{T}}^{\mathrm{exp}}\right\rangle\left[10^{-3}\right]$ & $\operatorname{Im}\left(x_{+}\right)\left[10^{-3}\right]$ \\
\hline background level & $\pm 10 \%$ & \pm 0.03 & \pm 0.2 \\
background asymmetry & $\pm 1 \%$ & \pm 0.02 & \pm 0.5 \\
$\xi$ & $\pm 4.3 \times 10^{-4}$ & \pm 0.2 & \pm 0.1 \\
$\eta$ & $\pm 2.0 \times 10^{-3}$ & \pm 1.0 & \pm 0.4 \\
decay-time resolution & $10 \%$ & negligible & \pm 0.6 \\
regeneration & Ref. $[8]$ & \pm 0.1 & \pm 0.1 \\
Total syst. & & \pm 1.0 & \pm 0.9 \\
\hline
\end{tabular}


$\pm 1.0 \times 10^{-3}$, respectively. As can be seen from Table 1 , the secondary vertex normalization $\eta$ is the dominant source of systematic error for our asymmetry.

The lifetime resolution for each lifetime bin was determined by a Monte Carlo simulation. Varying these resolutions within $\pm 10 \%$ we find a negligible change in $\left\langle A_{\mathrm{T}}^{\exp }\right\rangle$. The systematic errors arising from the error of $\Delta m, \Gamma_{\mathrm{S}}$ and $\Gamma_{\mathrm{L}}$ are negligible both for $\left\langle A_{\mathrm{T}}^{\exp }\right\rangle$ and $\operatorname{Im}\left(x_{+}\right)$.

Finally, the evaluation of the systematic error due to the regeneration effect is obtained by varying the value of the regeneration amplitude within its experimental errors. The resulting systematic error on $\left\langle A_{\mathrm{T}}^{\exp }\right\rangle$ is found to be $\pm 0.1 \times 10^{-3}$.

\section{Final results and conclusions}

The experimentally obtained asymmetry (see Section 4.4) is

$$
\left\langle A_{\mathrm{T}}^{\exp }\right\rangle=\left(6.6 \pm 1.3_{\text {stat }} \pm 1.0_{\text {syst }}\right) \times 10^{-3} \text {. }
$$

This value is well compatible with $4 \operatorname{Re}(\epsilon)$ (see Section 6). In the limit of CPT invariance in the semileptonic decay process but independently of the validity of the $\Delta S=\Delta Q$ rule, this is a departure from time-reversal invariance in the evolution of neutral kaons into their antiparticles. It constitutes the first direct measurement of a difference between the rate of a process and its inverse.

\section{Acknowledgements}

We would like to thank the CERN LEAR staff for their support and co-operation as well as the technical and engineering staff of our institutes. This work was supported by the following agencies: the French CNRS/Institut National de Physique Nucléaire et de Physique des Particules, the French Commissariat à l'Energie Atomique, the Greek General Secretariat of Research and Technology, the Netherlands Foundation for Fundamental Research on Matter (FOM), the Portuguese National Board for Science and Technology (JNICT), the Ministry of Science and Technology of the Republic of Slovenia, the Swedish Natural Science Research Council, the Swiss National Science Foundation, the UK Particle Physics and Astronomy Research Council (PPARC), and the US National Science Foundation.

\section{References}

[1] P.K. Kabir, Phys. Rev. D 2 (1970) 540; A. Aharony, Lett. Nuovo Cimento 3 (1970) 791.

[2] see, for instance, T.D. Lee, Particle Physics and Introduction to Field Theory (Harwood, Chur, 1981); R.G. Sachs, The Physics of Time Reversal (University of Chicago Press, Chicago, 1987) and references therein.

[3] R. Adler et al., CPLEAR Collaboration, Nucl. Instr. and Meth. A 379 (1996) 76

[4] Electron-muon-pion separation in the CPLEAR experiment using the Particle Identification Detector, Internal Note, CPLEAR/DET/95-02, January 1995.

[5] R. Adler et al., CPLEAR Collaboration, Phys. Lett. B 363 (1995) 237.

[6] R. Adler et al., CPLEAR Collaboration, Phys. Lett. B 363 (1995) 243.

[7] C. Caso et al., Particle Data Group, Eur. Phys. J. C 3 (1998) 1.

[8] A. Angelopoulos et al., CPLEAR Collaboration, Phys. Lett. B 413 (1997) 422.

[9] C.D. Buchanan et al., Phys. Rev. D 45 (1992) 4088.

[10] L. Maiani, CP and CPT violation in neutral kaon decays, in: L. Maiani et al. (Eds.), The Second DA $\Phi$ NE Physics Handbook, INFN, Frascati, 1995, p. 3. The parametrizations presented there are equivalent to ours but formulated in a slightly different notation. 